\title{
Modeling and solution based on stochastic games for development of COA under uncertainty
}

\author{
CHEN Chao ${ }^{1, *}$, DU Zhengjun ${ }^{2}$, LIANG Xingxing ${ }^{1}, \mathrm{SHI}$ Jianmai $^{1}$, and ZHANG Hao ${ }^{3}$ \\ 1. Science and Technology on Information Systems Engineering Laboratory, National University of Defense Technology, \\ Changsha 410073, China; \\ 2. Unit 61683 of the PLA, Beijing 100094, China; \\ 3. Naval War Gaming Center, Naval Command College, Nanjing 210016, China
}

\begin{abstract}
Developing a course of action (COA) is a key step in military planning. In most extant studies on the COA development, only the unilateral actions of friendly forces are considered. Based on stochastic games, we propose models that could deal with the complexities and uncertainties of wars. By analyzing the equilibrium state of both opponent sides, outcomes preferable to one side could be achieved by adopting the methods obtained from the proposed models. This research could help decision makers take the right $\mathrm{COA}$ in a state of uncertainty.
\end{abstract}

Keywords: course of action (COA), uncertainty of antagonism outcomes, stochastic games.

DOI: $10.21629 / J S E E .2019 .02 .08$

\section{Introduction}

The antagonism in an operation is fierce; both sides attempt to transform the initial situation to their own advantages, which is unquestionably contradictory [1]. The evolution of war greatly depends on the joint actions of both sides, which leads to the mutual influence on these actions [2-4]. This confrontation should be considered in planning a course of action (COA) [5]. The COA is utilized to optimize and organize actions that formulate plans under conditions of selectable operational actions of both sides, interrelationships between actions and situations, and resource constraints [6]. An information war is characterized by various operational elements, broad operations space, uncertainties, complicated relationships among operation elements, and the mutual influence actions arising from both sides [7,8]. Given the difficulty that a commander experiences in developing an operational plan from a series of feasible plans, he/she has to be

Manuscript received September 14, 2017.

*Corresponding author.

This work was supported by the Natural Science Foundation of China (71471174). provided with scientific and efficient decision-making supporting methods $[9,10]$.

Currently, studies on issues pertaining to the COA focus on operation research and artificial intelligence [1115]. By incorporating timing information, Wagenhals [16] transformed the model into a discrete event system model in the form of a Petri net, revealing the effect of the order and time of action events on the desired results and the effects of the situation. Tian [17] proposed a method based on a greedy algorithm to solve the optimal or near optimal operation process in the case of uncertainty [18], thereby allowing the user to observe the effect of an action on a desired effect. Recognizing that the core problem of the process of action is to choose the best combination of actions based on alternative action plans, Wan [19] established the COA modeling solution framework. The first module establishes the mobile network model, the second chooses the optimal plan from all the feasible combinations described in the first module, and the third tests the action process. Considering the conflict and resource constraints, Du [20] established a behavioral process model based on the sequence game theory which is more suited to confrontation and transformed it into a matrix game model. Sumile [21] outlined a target set of command and control (C2) modeling and simulation (M\&S) capabilities critical to providing commanders with adequate situational awareness (SA) to enable globally integrated operations. Sumile also provided a conceptual framework to formulate a collaborative information environment (CIE) that can support COA modeling and validation while planning during execution. In order to optimize the attack process against targets with complex relationships and highly dynamic features, Lei [22] described military targets and their relationships as a system of systems ( $\mathrm{SoS}$ ) which was modeled by using the dynamic bayesians network, and proposed a genetic algorithm based method to model the target-attacking 
COA under a resource constraint situation. Based on the discrete system modeling method, Haider et al. [23] proposed a formalism based on a timing influence network (TIN) to simulate the uncertainties in a dynamic context. Zaidi et al. [24] extended the TIN, extending the two aspects of time-varying and looping to expand the description of TIN. Haider et al. [25] used the TIN to model an uncertain situation, and an intelligent algorithm, e.g., particle swarm optimization and evolution, to identify the effective action process in that uncertain situation. Rafi et al. [26] used the activation TIN to describe the continuous evolution of an action and its environment, and in the case of a set of prerequisites, developed two algorithms to optimize these actions. Zaidi et al. [27] proposed a comprehensive theory of influence network, including consistency, time problems and design constraints that affect the dynamic evolution of constants. Based on classical artificial intelligence planning techniques and the principles of anytime and anywhere, Tang et al. [28] proposed a set of heuristic methods to select search nodes that take into account the preferences of emergency managers in the planning process. Considering the planning of military operations that historically focused on actions and their direct consequences, Pousi [29] studied the application of multi-criteria decision analysis (MCDA) to the planning of effects-based operations (EBOs). Yaman [30] developed a fuzzy cognitive map (FCM) to build EBOs, taking into account the constraints of problem characteristics and management. Considering the impact of conflict games, resource constraints, and execution time, Chen et al. [31] established a COA development model based on TINs and the game theory to solve a given problem by converting it into a standard matrix game model. In these studies, the influence of the actions of each side toward a war situation is considered and the transformation of the war situation is achieved by implementing those actions. However, the fact that targeting actions the enemy may choose could also affect the war situation remains unconsidered [32].

The counterparts of the red and the blue sides (hostile sides) are unlikely to obtain complete information and stochastic factors in one operation [33-35]. Hence, the transformation paths of a war situation are uncertain even if the current situation and actions from both sides are known, which we define as uncertainty of confrontation outcomes [36]. This could be largely explained by two aspects: on the one hand, accidental factors and stochastic events may cause this uncertainty; on the other hand, the information received from a commander is incomplete for making the right decision. To address the deficiencies in developing COAs, this study analyzes and works out solutions for uncertainties in antagonism outcomes by stochastic games models [37].

\section{Problem analysis}

War situations evolve under the joint action of both counterparts. Owing to the uncertainties resulting from stochastic factors [38], it is difficult to ensure war situations. Instead, estimating possible situations and their probabilities is possible. As shown in Fig. 1, in situation $S_{1}$, the red and blue sides take actions $\phi$ and $\psi$ respectively, and the war situation is transformed into $S_{2}, S_{3}$ in a corresponding probability of $p_{1}, p_{2}$.

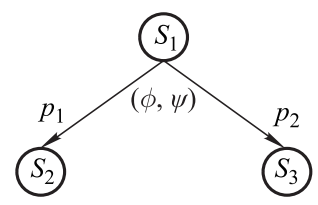

Fig. 1 Example of war situation transformation

The uncertainty of a war situation transformation makes it difficult for a commander to predict the transformation paths after he/she executes operational actions [38,39], and therefore the final outcome of the war is unknown and so is the decision-making effect of operational actions. According to the game theory, the uncertain situation of a confrontation outcome is actually the uncertainty of the system transformation for people inside the game. Thus, a stochastic-game model can be built to address the system transformation uncertainty that is described as a function of situation transformation.

\section{Model formulation}

\subsection{Model description}

\subsubsection{War situation}

A war situation includes information about the environment, disposition, and actions of both sides involved in an operational region. Actions from both sides have a direct influence on the situation, in which the foremost step in planning operational actions is to describe the situation, especially those significant elements which may influence the war situation. Drawing from cybernetics, a war situation is represented by the status variable $S$. Given that a war situation involves various elements, $S$ is rather intricate. $S$ could be a high-dimensioned function $S=$ $\left(s_{1}(t), s_{2}(t), \ldots, s_{m}(t)\right)$ related to time. Appropriate operational actions require reasonably simplified variables according to real-time conditions and refined key elements that may affect the operation actions, wherein the refined elements of the war situation should be discrete. Meanwhile, the war situation for each phase divided by an operational section can be represented by a multi-dimensional vector. Each element in the vector denotes a factor that may exert influence on a war situation. The transformation 
of war situation is determined by the operational actions from both sides, which can be described as consumption of the operational resources and changes in military arrangement. The entire operational action is divided into $K$ sections with the initial status $S_{1}$, and possible action sets for the red and the blue sides being $\Phi_{1}, \Psi_{1}$. After implementing possible actions $\phi_{i}^{1}$ and $\psi_{j}^{1}$ from the sets, the situation may be transformed into several situations $\left\{{ }_{i j} S_{m_{i j}}^{2}\right\}_{m_{i j}=1}^{M_{i j}}$ in the next section.

As shown in Fig. 2 , in the $k$ th $(1 \leqslant k \leqslant K)$ section, after adopting actions $\left(\phi_{i}^{k}, \psi_{j}^{k}\right)$ from both the red and the blue sides, the situation for the $(k+1)$ th section could be transformed into several possible resultant situations. The transformation can be illustrated as a tree diagram $T=(S, E)$, where $S$ is the nodes set representing different war situations, and $E$ is the linking edges set denoting system transformation under the actions of both the red and the blue sides. Linking edge $\left\{\left(\phi_{i}, \psi_{j}\right), p_{l}\right\}$ represents a transformation into a target situation with a probability of $p_{l}$ under the action $\left(\phi_{i}, \psi_{j}\right)$.

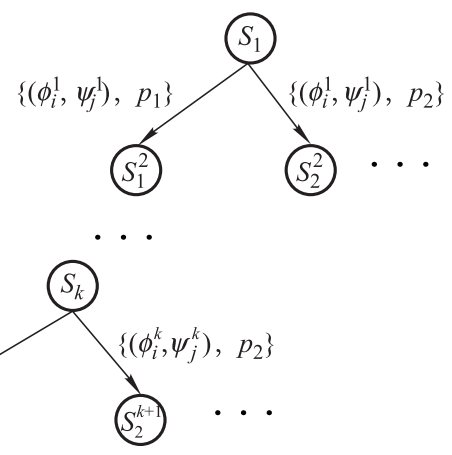

Fig. 2 War situation transformation in an operation

\subsubsection{Action}

Action is a decisive variable for a decision maker in a game, as well as a basic element for a game. In order to distinguish the term "action" from the theory of games, the basic operational actions for the red and the blue sides are defined as "tasks". In each section of the operation, both the red and the blue sides have a set of tasks that can be adopted. In section $k$, the red side has $h_{k}$ selectable tasks $\left\{r t_{1}^{k}, \ldots, r t_{h_{k}}^{k}\right\}$, while the blue side has $l_{k}$ selectable tasks $\left\{b t_{1}^{k}, \ldots, b t_{l_{k}}^{k}\right\}$. In this study, the decisive variable for both the red and the blue sides in a section is the task vector, which meets the resource requirement and action constraints. Therefore, the action is defined as binaryrealization of the task vector. In the situation $S_{k}$, the action set of the red side is denoted by $\Phi\left(S_{k}\right)$, and abbreviated as $\Phi_{k}$, and $\Phi^{(k)}=\left\{\phi_{1}^{(k)}, \phi_{2}^{(k)}, \ldots, \phi_{f_{k}}^{(k)}\right\}$. In addition, the action set of the blue side is denoted by $\Psi\left(S_{k}\right)$ and abbreviated as $\Psi_{k}$. Furthermore, the possible actions for both sides need to be analyzed and decided by military experts in accordance with real-life situations.

\subsubsection{War situation transformation probability}

The war situation transformation is co-affected by both the red and the blue sides, with a certain uncertainty which is described by transformation probability. With the situation $S_{k}$ and the actions $\phi_{i}^{k}, \psi_{j}^{k}$ defined above, the probability for system transformation situation $S^{\prime}$ is expressed as $P_{\psi_{j}^{k}, \phi_{i}^{k}}\left(S^{\prime} \mid S_{k}\right)$, where $\sum_{S^{\prime} \in S_{k, \phi_{i}^{k}, \psi_{j}^{k}}} P_{\psi_{j}^{k}, \phi_{i}^{k}}\left(S^{\prime} \mid S_{k}\right)=1$. $S_{k, \phi_{i}^{k}, \psi_{j}^{k}}$ denotes the set of possible system situation transformations after the system $S_{k}$ has experienced actions $\phi_{i}^{k}, \psi_{j}^{k}$ from both the red and the blue sides. The probability can be estimated by military experts, either from prior experience or from operational experiments. Similar to the transformation probability described above, we can further redefine the action sets of both the red and the blue sides. The action set for the red side is defined as $\Phi\left(S_{k}\right)=\left\{\phi_{i}^{k} \in \Phi_{k} \mid \exists S^{\prime} \in S, P_{\psi_{j}^{k}, \phi_{i}^{k}}\left(S^{\prime} \mid S_{k}\right) \neq 0\right\}$, while that of the blue side is defined as $\Psi\left(S_{k}\right)=\left\{\psi_{j}^{k} \in\right.$ $\left.\Psi_{k} \mid \exists S^{\prime} \in S, P_{\psi_{j}^{k}, \phi_{i}^{k}}\left(S^{\prime} \mid S_{k}\right) \neq 0\right\}$.

\subsubsection{Strategy}

There exists an effect of mutual dependence between the actions of both sides and the war situation. The latter determines the actions of both sides, while the actions from both sides determine the transformation path of the war situation. Therefore, the strategy should reflect real conditions, namely a strategy offering action policies corresponding to all situations for the player. Strategy is defined as the reflection of the action set $\Phi, \Psi$ from the situation $S$, which is denoted by $\pi_{r}: S \rightarrow \Phi, \pi_{b}: S \rightarrow \Psi$.

$$
\Phi=\left\{\Phi_{1}, \Phi_{2}, \ldots, \Phi_{M}\right\}, \Psi=\left\{\Psi_{1}, \Psi_{2}, \ldots, \Psi_{M}\right\}
$$

represent the sets of possible actions for the red and the blue sides in situation $m(1 \leqslant m \leqslant M)$.

Vectors $\boldsymbol{\pi}_{r}^{i}=\left(\phi_{i_{1}}^{1}, \phi_{i_{2}}^{2}, \ldots, \phi_{i_{m}}^{m}, \ldots, \phi_{i_{M}}^{M}\right), \boldsymbol{\pi}_{b}^{j}=$ $\left(\psi_{j_{1}}^{1}, \psi_{j_{2}}^{2}, \ldots, \psi_{j_{m}}^{m}, \ldots, \psi_{j_{M}}^{M}\right)$ stand for the $i$ th and $j$ th strategies of the red and the blue sides respectively, where the action $\phi_{i_{m}}^{1}$ of the red side and $\psi_{j_{m}}^{m}$ of the blue side correspond to the $i$ th and $j$ th strategies in the situation $S_{m}$.

\subsubsection{Effect function}

Effect function of the red and the blue sides is the function for the final war situation. Both sides expect the final situation to be in line with their initial expectations. Therefore, there may exist a preferred ranking for the possible confrontation outcomes. Besides, the counterparts in a war have antagonistic targets for operations, suggesting that the victory of one side represents failure for the other. There- 
fore, we define the game model between the two sides as a zero-sum game with the red side as the decision-maker of Max type, and the blue side as the decision-maker of the Min type. Effect function stands for the various possible final situations, where the possible $N_{K}$ situations at the final section constitute vector $\boldsymbol{S}_{N_{K}}$ and the effect function $f\left(\boldsymbol{S}_{N_{K}}\right) \rightarrow \Re$ should be determined by military experts.

\subsection{Mathematical model}

In summary, with the uncertainty of the confrontational outcome, the development of the COA can be modeled as a stochastic game, which can be described by a quintuple, $\Gamma=(N, S, \Omega, P, u)$.

i. $N=\{R, B\}$ denotes the red (R) and blue (B) sides in the game;

ii. $S=\left\{S_{1}, S_{2}, \ldots, S_{M}\right\}$ denotes the set of the war situation;

iii. $\Omega=\{\Phi, \Psi\}$ denotes the set for the actions of both sides;

iv. $\Phi=\left\{\Phi_{1}, \Phi_{2}, \ldots, \Phi_{M}\right\}$, and $\Phi_{m}$ denotes the set of feasible actions of the red side;

v. $\Psi=\left\{\Psi_{1}, \Psi_{2}, \ldots, \Psi_{M}\right\}$, and $\Psi_{m}$ denotes the set of feasible actions of the blue side;

vi. $P$ denotes the probability of the war situation transformation;

vii. $P_{\psi_{j}^{k}, \phi_{i}^{k}}\left(S_{l} \mid S_{k}\right)$ denotes the probability that the red and the blue sides are in the situation $S_{k}$ and have executed actions $\phi_{i}^{k}, \psi_{j}^{k}$, respectively;

viii. $U$ denotes the effect function;

ix. $u=f\left(\boldsymbol{S}_{N_{k}}\right)$ denotes the effect function of the red side.

\section{Definition and solution to a balanced strategy}

\subsection{Nature for equilibrium strategy}

\subsubsection{Definition of equilibrium}

Owing to the mutual effect of actions from both the red and the blue sides, a rational decision maker would search for an equilibrium strategy for his/her own side and set it as his/her decision-making criterion [40]. The COA models with uncertainty of the confrontation outcome are $\boldsymbol{\pi}_{r}^{i}, \boldsymbol{\pi}_{b}^{j}$, and the mixed strategies are $\sigma_{r}^{i}, \sigma_{b}^{j}$.

Definition 1 The equilibrium strategy $\left(\sigma_{r}^{*}, \sigma_{b}^{*}\right)$ has the following requirements.

i. For the red side, the equilibrium strategy $\sigma_{r}^{*}$ has the following requirements: $u\left(\sigma_{r}^{*}, \sigma_{b}^{*}\right) \geqslant u\left(\sigma_{r}^{k}, \sigma_{b}^{*}\right), \sigma_{r}^{k} \in$ $\Delta_{r}, \Delta_{r}$ is the mixed strategy for the red side.

ii. For the blue side, the equilibrium strategy $\sigma_{b}^{*}$ has the following requirements: $u\left(\sigma_{r}^{*}, \sigma_{b}^{*}\right) \leqslant u\left(\sigma_{r}^{*}, \sigma_{b}^{n}\right), \sigma_{b}^{n} \in$ $\Delta_{b}, \Delta_{b}$ is the mixed strategy for the blue side.

\subsubsection{Existence for an equilibrium}

Statement 1 Given the stochastic games $\Gamma=(N, S$, $\Omega, P, U)$, an equilibrium solution exists if the action set and the situation set of the war are finite sets [41].

The strategy set of the red side is $\pi_{r}$, as the situation set $S=\left\{S_{1}, S_{2}, \ldots, S_{M}\right\}$ is finite, as well as an action set $\Phi_{m}(1 \leqslant m \leqslant M)$ in any war situation. Therefore, the pure strategy set $\pi_{r}^{i}=\left\{\pi_{r}^{1}, \pi_{r}^{2}, \ldots, \pi_{r}^{i}, \ldots, \pi_{r}^{I}\right\}$ for the red side is a finite set. Similarly, the pure strategy set $\boldsymbol{\pi}_{b}^{j}=\left\{\pi_{b}^{1}, \pi_{b}^{2}, \ldots, \pi_{b}^{j}, \ldots, \pi_{b}^{J}\right\}$ is also finite. For the mixed strategy set $\sigma_{r}^{k}$ of the red side, we define the probability of the pure strategy $\pi_{r}^{i}(1 \leqslant i \leqslant I)$ as $p_{k}^{i}$, where $0 \leqslant p_{k}^{i} \leqslant 1$ and $\sum_{i=1}^{I} p_{k}^{i}=1$, and the mixed strategy $\sigma_{r}^{k}$ is degraded into the pure strategy $\boldsymbol{\pi}_{r}^{i}$ if $p_{k}^{i}=1$. When the mixed strategy for the blue side is $\sigma_{b}^{n}$, we decide the probability of the pure strategy $\pi_{b}^{j}(1 \leqslant j \leqslant J)$ as $q_{n}^{j}$, $0 \leqslant q_{n}^{j} \leqslant 1$, and $\sum_{j=1}^{J} q_{n}^{j}=1$, when $q_{n}^{j}=1$ the mixed strategy is degraded into the pure strategy $\boldsymbol{\pi}_{b}^{j}$. When the red and the blue sides adopt the pure strategies $\boldsymbol{\pi}_{r}^{i}$ and $\boldsymbol{\pi}_{b}^{j}$, respectively, the earned value of the red side can be calculated by effect function $a_{i j}$, and the earned value of the blue side is $-a_{i j}$ owing to the nature of the zero-sum game. When the red and the blue sides adopt the mixed strategies $\sigma_{r}^{k}$ and $\sigma_{b}^{n}$, the expected return for the red side is $\sum_{j=1}^{J} \sum_{i=1}^{I} p_{k}^{i} q_{n}^{j} a_{i j}$, and that of the blue side is $-\sum_{j=1}^{J} \sum_{i=1}^{I} p_{k}^{i} q_{n}^{j} a_{i j}$. We define set-valued function $F: \Delta \rightarrow \Delta$, where $\Delta$ denotes the Descartes product by $\Delta_{r}$ and $\Delta_{b}$. In addition, $\sigma=\left(\sigma_{r}, \sigma_{b}\right)$ denotes the strategy combination of the both side. For any mixed strategy $\sigma_{b}^{n}, F$ will make the red side to select its own strategy $\sigma_{r}^{k}$ to maximize the expected return $u_{k n}=\sum_{j=1}^{J} \sum_{i=1}^{I} p_{k}^{i} q_{n}^{j} a_{i j}$, namely $\underset{p_{k}^{i}}{\arg \max } \sum_{i=1}^{I} \sum_{j=1}^{J} q_{n}^{j} a_{i j}$. Furthermore, for any mixed strategy $\sigma_{r}^{k}, F$ will make the blue side to select its own strategy $\sigma_{b}^{n}$ to maximize the expected return $u_{n k}=-\sum_{j=1}^{J} \sum_{i=1}^{I} p_{k}^{i} q_{n}^{j} a_{i j}$, namely $\underset{q_{n}^{j}}{\arg \min } \sum_{i=1}^{I} \sum_{j=1}^{J} p_{k}^{i} a_{i j}$

To prove the equilibrium in the model $\Gamma=(N, S, \Omega$, $P, U)$, we need to prove that there exists a fixed point in $F: \Delta \rightarrow \Delta$, namely, it has to meet the requirement of the Kakutani theorem. Besides, the earned value of the players is a linear function of its mixed strategy, and therefore 
the mixed strategy is consecutive. To verify that the situation meets the requirements of the Kakutani theorem is to testify the following conditions:

(i) $F(\sigma)$ is convex.

In order to maximize the expected return $\sum_{j=1}^{J} \sum_{i=1}^{I} p_{k}^{i} q_{n}^{j} a_{i j}$ of the red side, we define $u_{i}=\sum_{j=1}^{J} q_{n}^{j} a_{i j}$ and $u=\sum_{i=1}^{I} p_{k}^{i} u_{i}$. In order to maximize $u, p_{k}$ must belong to the following set:

$$
P=\left\{p_{k}: p_{k}^{i} \geqslant 0, \sum p_{k}^{i}=1, \text { if } u_{i}<\max _{t} u_{t}, p_{k}^{i}=0\right\}
$$

namely $p_{k} \in P\left(q_{n}\right)$.

Similarly, for the blue side, in order to maximize its expected return, $q_{n}$ must belong to the following set:

$$
Q=\left\{q_{n}: q_{n}^{j} \geqslant 0, \sum q_{n}^{j}=1, \text { if } u_{j}<\max _{t} u_{t}, q_{n}^{j}=0\right\},
$$

namely $q_{n} \in P\left(p_{k}\right)$.

As $\boldsymbol{F}$ stands for the optimal response function for strategies of both the red and the blue sides, it has the following nature:

$$
\boldsymbol{F}(\boldsymbol{\sigma})=\left(\begin{array}{l}
P(q) \\
Q(p)
\end{array}\right) .
$$

Obviously, $\boldsymbol{F}(\boldsymbol{\sigma})$ is convex.

(ii) $\boldsymbol{F}(\boldsymbol{\sigma})$ has upper semi continuity, for

$$
\widehat{\boldsymbol{\sigma}^{n}} \in \boldsymbol{F}\left(\boldsymbol{\sigma}^{n}\right),\left(\boldsymbol{\sigma}^{n}, \widehat{\boldsymbol{\sigma}^{n}}\right) \rightarrow(\boldsymbol{\sigma}, \widehat{\boldsymbol{\sigma}}), \widehat{\boldsymbol{\sigma}} \in \boldsymbol{F}(\boldsymbol{\sigma}) .
$$

Set $\boldsymbol{\sigma}^{n}=\left(\begin{array}{c}p^{n} \\ q^{n}\end{array}\right), \widehat{\boldsymbol{\sigma}^{n}}=\left(\begin{array}{c}w^{n} \\ v^{n}\end{array}\right)$.

As $\widehat{\boldsymbol{\sigma}^{n}} \in \boldsymbol{F}\left(\boldsymbol{\sigma}^{n}\right)$, namely, $\widehat{\boldsymbol{\sigma}^{n}}$ is the optimal response for $\boldsymbol{\sigma}^{n}$.

Therefore, for any strategy $p^{\prime}$ of the red side,

$$
u\left(w^{n}, q^{n}\right)>u\left(p^{\prime}, q^{n}\right) .
$$

And for any strategy $q^{\prime}$,

$$
u\left(p^{n}, v^{n}\right)>u\left(p^{n}, q^{\prime}\right) .
$$

When $\boldsymbol{\sigma}^{n} \rightarrow \boldsymbol{\sigma}, \widehat{\boldsymbol{\sigma}^{n}} \rightarrow \widehat{\boldsymbol{\sigma}}$, we can obtain $p^{n} \rightarrow p, q^{n} \rightarrow$ $q, w^{n} \rightarrow w, v^{n} \rightarrow v$.

Then, for any strategy $p^{\prime}$ of the red side, $u(w, q)>$ $u\left(p^{\prime}, q\right)$. Namely, $w$ is the optimal response for $q$. For any strategy $q^{\prime}$ of the blue side, $u(p, v)>u\left(p, q^{\prime}\right)$. Namely, $v$ is the optimal response for $p$.

$$
\widehat{\boldsymbol{\sigma}}=\left(\begin{array}{c}
w \\
v
\end{array}\right) \text { is the optimal response for } \boldsymbol{\sigma}=\left(\begin{array}{l}
p \\
q
\end{array}\right) \text {, }
$$
namely $\widehat{\boldsymbol{\sigma}} \in \boldsymbol{F}(\boldsymbol{\sigma})$.
Conditions (i) and (ii) hold true, and therefore, the stochastic game model $\Gamma=(N, S, \Omega, P, U)$ for the COA exists when the antagonism outcome is uncertain.

\subsubsection{Refinement of balance}

Statement 1 explains the existence of an equilibrium solution for the model. However, owing to the incredible balance caused by the transformation from stochastic games into strategic games, we need to analyze which balance is rational. In order to facilitate the analysis of the equilibrium of the game, we expand the general game tree diagram by adding chance nodes to describe the uncertainty of situation transformation, where " $\diamond$ " denotes the chance nodes as shown in Fig. 3.

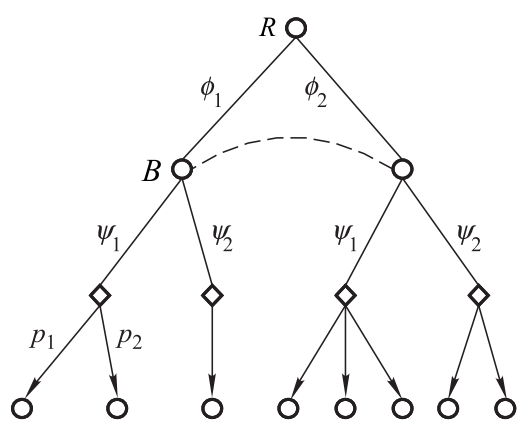

Fig. 3 Tree diagram of stochastic games

Nodes in the tree are divided into decisive nodes and chance nodes. The former stands for the position wherein a decision maker locates when he/she needs to make a decision, while the latter represents the uncertainty of war situation transformation or possible situation nodes. Linking edge starting with a decisive node stands for action of the players (such as $\phi_{1}, \phi_{2}$ ); linking edge starting with a chance node stands for the probability of situation transformation under actions from both sides (such as $p_{1}, p_{2}$ ). With the premise of uncertain confrontation outcomes, the stochastic game of the red and the blue sides takes on the nature of an expanded game. In an expanded game, the transformation from the expanded game to a strategic game is an "incredible threat," and a refinement for the balance is subsequently required. Currently, the most acceptable concept of equilibrium is a subgame perfect equilibrium, which requires every proper subgame to represent the Nash equilibrium balance [42]. We define a proper subgame and a subgame perfect equilibrium according to the definitions of proper subgames and subgame perfect equilibrium from expanded games.

Definition 2 The proper subgame of a stochastic game is defined as a tree that contains structures starting with situation nodes, followed by the sequencing nodes and edges linking those nodes. 
Definition 3 The perfect Nash equilibrium of the subgame requires that each anatomical side of the equilibrium strategy should constitute a Nash equilibrium in each proper subgame.

A subgame perfect equilibrium in stochastic games proposes a reasonable equilibrium after a stochastic game has been transformed into a matrix game. We need to analyze the condition of the perfect subgame based on its definition, which lays the foundation for its solution.

Statement 2 A subgame perfect equilibrium in a stochastic game $\Gamma=(N, S, \Omega, P, U)$ is a Nash equilibrium from the root nodes to the leaf nodes.

$(\Rightarrow)$ suppose that there exists a subgame perfect equilibrium that does not contain a full path, then there must be a node that fails to lead the nodes following it to the path of the balanced strategy alongside the path directing it to its destination. This is contrary to the definition of the subgame perfect equilibrium, and thus the hypothesis should be rejected. Therefore, there does not exist a subgame perfect equilibrium that does not contain a full path.

Statement 2 proposes a solution to a subgame perfect equilibrium with the uncertainty of confrontation outcome, which is to work out the equilibrium by a transformation into a matrix game and analyze if the solution constitutes a path that links the root to the final sub-nodes of a game tree structure.

\subsection{Solution to a balanced strategy}

According to the definitions of strategy and effect function, we can obtain a strategy $\boldsymbol{\pi}_{r}^{i}$ of both sides and a strategy $\pi_{b}^{j}$ of the blue side. The payment value of both sides is $u\left(\boldsymbol{\pi}_{r}^{i}, \boldsymbol{\pi}_{b}^{j}\right)$. The payment matrix is then calculated and so is the balanced strategy for both sides. The specific solutions are as follows:

(i) The strategy sets of the red and blue sides $\pi_{r}, \pi_{b}$, respectively, are determined according to the situation set $S$ and actions set $\Omega$.

In addition, the $i$ th strategy of the red side is $\boldsymbol{\pi}_{r}^{i}=$ $\left(\phi_{i_{1}}^{1}, \phi_{i_{2}}^{2}, \ldots, \phi_{i_{m}}^{m}, \ldots, \phi_{i_{M}}^{M}\right)$, which denotes the action $\phi_{i_{m}}^{1}$ that the red side adopts in the system situation $S_{m}$. The $j$ th strategy of the blue side is $\pi_{b}^{j}=$ $\left(\psi_{j_{1}}^{1}, \psi_{j_{2}}^{2}, \ldots, \psi_{j_{m}}^{m}, \ldots, \psi_{j_{M}}^{M}\right)$, which denotes the action $\psi_{j_{m}}^{m}$ in the system situation $S_{m}$.

(ii) The payment matrix is determined according to the set of strategies and effect functions.

For the $i$ th strategy of the red side and the $j$ th strategy of the blue side, the probability of the final situation can be determined according to the probability of situation transformation, and subsequently, its payment value can be determined according to the effect function, and the entire payment matrix can be determined in a similar way. (iii) The balanced strategy and the earned value of the red and the blue sides can be determined by the payment matrix.

After the payment has been determined, it is easy to work out balanced strategies for both sides and their earned values.

(iv) Analyze the balanced strategy to work out the subgame perfect equilibrium.

Analyze if the equilibrium strategy contains the full path starting from the root nodes to the leaf nodes. The strategy containing the full path is known as a subgame perfect equilibrium.

\section{Computational experiments}

\subsection{Example design}

Suppose that an operation is divided into two sections, and the initial section is $S_{0}$. In $S_{0}$, the red and the blue sides can adopt actions $\phi_{1}^{0}, \phi_{2}^{0}$ and $\psi_{1}^{0}, \psi_{2}^{0}$ respectively. The detailed numerical example is shown in Fig. 4. After the adoption of $\phi_{1}^{0}$ and $\psi_{2}^{0}$, the system situation will be transformed into $S_{2}$. After the adoption of $\phi_{2}^{0}$ and $\psi_{1}^{0}$, the system situation will be transformed into $S_{3}$; after the adoption of $\phi_{2}^{0}$ and $\psi_{2}^{0}$, the system situation will be transformed into $S_{4}$ with a probability of 0.3 , and into $S_{5}$ with a probability of 0.7 . In $S_{1}$, the red and the blue sides can adopt actions $\phi_{1}^{1}$ and $\psi_{1}^{1}$, and subsequently, the system situation will be transformed into $S_{6}$ and $S_{7}$ with a probability of 0.5 respectively. In $S_{2}$, the red and the blue sides can adopt actions $\phi_{1}^{2}$ and $\psi_{1}^{2}$, and the system situation will be transformed into $S_{8}$ and $S_{9}$ with probabilities of 0.4 and 0.6 , respectively. In $S_{3}$, the red side can adopt actions $\phi_{1}^{3}$ and $\phi_{2}^{3}$, and the blue side can adopt $\psi_{1}^{3}$. Following the adoption of $\phi_{1}^{3}$ and $\psi_{1}^{3}$, the system will be transformed into $S_{10}$; after the adoption of $\phi_{2}^{3}$ and $\psi_{1}^{3}$, the system situation will be transformed into $S_{11}$ and $S_{12}$ with probabilities of 0.8 and 0.2 respectively.

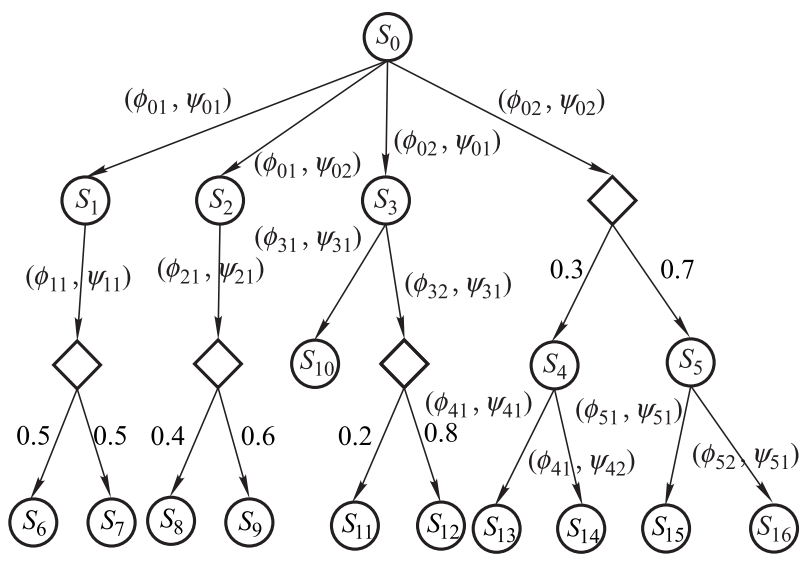

Fig. 4 Example of a stochastic game

In $S_{4}$, the red side can adopt action $\phi_{1}^{4}$ while the blue 
side adopts $\psi_{1}^{4}$ and $\psi_{2}^{4}$. Following the adoption of $\phi_{1}^{4}$ and $\psi_{1}^{4}$, the system will be transformed into $S_{13}$; after the adoption of $\phi_{1}^{4}$ and $\psi_{2}^{4}$, the system will be transformed into $S_{14}$. In $S_{5}$, the red side can adopt actions $\phi_{1}^{5}$ and $\phi_{2}^{5}$, and the blue side $\psi_{1}^{5}$. After the adoption of $\phi_{1}^{5}$ and $\psi_{1}^{5}$, the system will be transformed into $S_{15}$; after the adoption of $\phi_{2}^{5}$ and $\psi_{1}^{5}$, the system situation will be transformed into $S_{16}$.

\subsection{Example solution}

The strategy sets for both sides are determined according to the action set of the situation set.

In $S_{0}, S_{3}$, and $S_{5}$, two actions can be adopted by the red side, whereas only one action can be adopted in $S_{1}, S_{2}$ and $S_{4}$, resulting in eight strategies for the red side. In $S_{0}$ and $S_{4}$, the blue side can adopt two actions, and the red side can only adopt one action in $S_{1}, S_{2}, S_{3}$, and $S_{5}$, resulting in four strategies for the blue side. Strategy is the reflection from an action space into a situation space. For instance, if the red side chooses actions $\phi_{1}^{0}, \phi_{1}^{1}, \ldots, \phi_{1}^{5}$ in situation $S_{0}, S_{1}, \ldots, S_{5}$ respectively, then the strategy is described as $(1,1,1,1,1,1)$, which is the first action in every situation. The strategy of the blue side is described in the same way.

Hence, the strategy sets of the red side are as follows:

$$
\begin{gathered}
\{(1,1,1,1,1,1),(1,1,1,1,1,2),(1,1,1,2,1,1), \\
(2,1,1,1,1,1),(1,1,1,2,1,2),(2,1,1,1,1,2), \\
(2,1,1,2,1,1),(2,1,1,2,1,2)\} .
\end{gathered}
$$

The strategy sets of the blue side are as follows:

$$
\begin{aligned}
& \{(1,1,1,1,1,1),(1,1,1,1,2,1), \\
& (2,1,1,1,1,1),(2,1,1,1,2,1)\} .
\end{aligned}
$$

The payment matrix is determined according to the strategy set and the effect function. The details are shown in Table 1.

Table 1 Payment matrix of the red side

\begin{tabular}{ccccc}
\hline & $(1,1,1,1,1,1)$ & $(1,1,1,1,2,1)$ & $(2,1,1,1,1,1)$ & $(2,1,1,1,2,1)$ \\
\hline$(1,1,1,1,1,1)$ & 5 & 5 & 4.8 & 4.8 \\
$(1,1,1,1,1,2)$ & 5 & 5 & 4.8 & 4.8 \\
$(1,1,1,2,1,1)$ & 5 & 5 & 4.8 & 4.8 \\
$(2,1,1,1,1,1)$ & 7 & 7 & 1.6 & 2.1 \\
$(1,1,1,2,1,2)$ & 5 & 5 & 4.8 & 4.8 \\
$(2,1,1,1,1,2)$ & 7 & 7 & 7.9 & 8.5 \\
$(2,1,1,2,1,1)$ & 10.4 & 10.4 & 1.6 & 2.1 \\
$(2,1,1,2,1,2)$ & 10.4 & 10.4 & 7.9 & 8.5 \\
\hline
\end{tabular}

Calculating the balanced strategy, we obtain the equilibrium strategy for the red side $(2,1,1,2,1,2)$, and that of the blue $(2,1,1,1,1,1)$. The earned value for the red side is 7.9, suggesting that in $S_{0}$, the red side adopts $\phi_{2}^{0}$ and the blue side adopts $\psi_{2}^{0}$; if the situation is transformed into $S_{4}$, then the red side adopts $\phi_{1}^{4}$ and the blue side adopts $\psi_{1}^{4}$; if the situation is transformed into $S_{5}$, then the red side adopts $\phi_{2}^{5}$ and the blue side adopts $\psi_{1}^{5}$.

$$
\phi_{2}^{\prime}(n, m) \phi_{3}^{\prime}(n, k) \psi(n, l)
$$

\section{Conclusions}

War is accompanied by fierce confrontation, and the evolution of the battlefield situation depends on the joint actions of both sides. Hence the strategies and correctness of their actions are closely interrelated. Under the condition of uncertainty of the confrontation outcome, we build a stochastic-game-based model to develop a COA and describe the operation action sequence from the aspects of the war situation, actions, situation transformation, and effect function. We propose the definition of equilibrium and analyze the existence of a balanced solution, as well as the conditions for a subgame perfect equilibrium. Moreover, we also propose a solution to it. Based on this work, we put forward an example and explain its solution for application.

\section{References}

[1] GUI J, ZHOU K. Flexible adjustments between energy and capacity for topology control in heterogeneous wireless multihop networks. Journal of Network and Systems Management, 2016, 24(4): 789-812.

[2] WU ZZ, ZHUANG Y. Linear-quadratic partially observed forward - backward stochastic differential games and its application in finance. Applied Mathematics \& Computation, 2018, 321: $577-592$.

[3] ZHANG W, WANG B, CHEN D. Continuous-time constrained stochastic games with average criteria. Operations Research Letters, 2017, 46(1): 109-115.

[4] SÈVE C, LEBLANC S, DURAND M, et al. Course-of-action theory in table tennis: a qualitative analysis of the knowledge used by three elite players during matches. Revue Europeenne de Psychologie Appliquee, 2017, 55(3): 145-155.

[5] ZILIOTTO B. Tauberian theorems for general iterations of operators: applications to zero-sum stochastic games. Games \& Economic Behavior, 2018: 1-18.

[6] ALlAMIGEON X, GAUBERT S, SKOMRA M. Solving generic nonarchimedean semidefinite programs using stochastic game algorithms. Journal of Symbolic Computation, 2018, 85: $25-54$

[7] ALTMAN E, PELLEGRINI F D. Dynamic games in novel networks: guest editors' forewords. Dynamic Games \& Applications, 2016, 6(4): $427-428$

[8] BAGH A. Existence of equilibria in constrained discontinuous games. International Journal of Game Theory, 2016, 45(4): $769-793$.

[9] BENSOUSSAN A, DELARUE F. Editorial: first issue on mean field games. Applied Mathematics \& Optimization, 2016, 74(3): 455-457.

[10] BERTRAND P, MUHAREMOVIC T, JIANG J. Random access design for high doppler in wireless networks. U.S. Patent 8,199,706. 2012-6-12.

[11] SHEN J, FENG D. A game-theoretic method for cross-layer stochastic resilient control design in CPS. International Jour- 
nal of Systems Science, 2018, 49(4): 677-691.

[12] SUN J, YONG J. Linear quadratic stochastic two-person nonzero-sum differential games: open-loop and closed-loop Nash equilibria m. Stochastic Processes \& Their Applications, 2019, 129(2): $381-418$.

[13] LAMPRECHT A L, TURNER K J. Scientific workflows. International Journal on Software Tools for Technology Transfer, 2016, 261(2): $1357-1398$.

[14] LUIRO H, PARVIAINEN M. Regularity for nonlinear stochastic games. Annales de Linstitut Henri Poincaré C Analyse Non Linéaire, 2018, 42(5): $2161-2196$.

[15] LV J, RONG J. Virtualisation security risk assessment for enterprise cloud services based on stochastic game nets model. IET Information Security, 2018, 12(1): 7-14.

[16] WAGENHALS L W, LEVIS A H. Course of action development and evaluation. Fairfax, Virginia, US: System Architectures Laboratory of George Mason University, 2000.

[17] TIAN Z, LI J X. Approach for course of actions determination in influence nets based on greedy algorithm. Command Control \& Simulation, 2013, (3): 14 - 17. (in Chinese)

[18] WANG Y, WANG L. Forward backward stochastic differential games for optimal investment and dividend problem of an insurer under model uncertainty. Applied Mathematical Modelling, 2018, 58: 254-269.

[19] WAN L, LI W, HUANG A. The modeling frame and generation flow of combat course of action. Proc. of the 5th International Conference on Frontiers of Manufacturing Science and Measuring Technology, 2017: 1621-1627.

[20] DU Z J, CHEN C, JIANG X. Modeling and solution of course of action based on influence net and sequential game. Systems Engineering-Theory \& Practice, 2013, 33(1): 215-222. (in Chinese)

[21] SUMILE M S. Collaborative modeling \& tailored simulation for course of action validation. Proc. of the Military Modeling \& Simulation Symposium, 2013: No. 1.

[22] LEI T, ZHU C, ZHANG W. Dynamic Bayes network based target-attacking course of action generation. Proc. of the IEEE International Conference on Signal Processing, Communication and Computing, 2013: 1-5.

[23] HAIDER S, LEVIS A H. Modeling time-varying uncertain situations using dynamic influence nets. International Journal of Approximate Reasoning, 2008, 49(2): 488-502.

[24] ZAIDI A K, LEVIS A H, PAPANTONI-KAZAKOS P. On extending temporal models in timed influence networks. Fairfax, Virginia, US: System Architectures Laboratory of George Mason University, 2009.

[25] HAIDER S, LEVIS A H. Finding effective courses of action using particle swarm optimization. Proc. of the Evolutionary Computation, 2008: 1135-1140.

[26] RAFI M F, ZAIDI A K, LEVIS A H, et al. Optimization of actions in activation timed influence nets. Informatica, 2009, 33(3): $285-296$.

[27] ZAIDI A K, MANSOOR F, PAPANTONI-KAZAKOS T P. Theory of influence networks. Journal of Intelligent \& Robotic Systems, 2010, 60(3-4): 457-491.

[28] TANG P, WANG H, QI C. Anytime heuristic search in temporal HTN planning for developing incident action plans. Artificial Intelligence Communications, 2012, 25(4): 321-342.

[29] POUSI J. Decision analytical approach to effects-based operations. Helsinki, Finland: Helsinki University of Technology, 2009.

[30] YAMAN D. A fuzzy cognitive map approach for effect-based operations: an illustrative case. Information Sciences, 2009, 179(4): $382-403$.
[31] CHEN C, KONG D F, DU Z J, et al. Modeling and solution of COA development based on timed influence net and game theory. Applied Mathematical Modelling, 2014, 38(21-22): $5269-5278$.

[32] WEI L, SARWAT A, SAAD W, et al. Stochastic games for power grid protection against coordinated cyber-physical attacks. IEEE Trans. on Smart Grid, 2018, 9(2): 684-694.

[33] FENG X. Stochastic differential games with competing Brownian particles and related Isaacs' equations. Optimal Control Applications \& Methods, 2018, 39(2): 519-536.

[34] WANG Y, WANG L. Forward-backward stochastic differential games for optimal investment and dividend problem of an insurer under model uncertainty. Applied Mathematical Modelling, 2018, 58: 254-269.

[35] SHEN J, FENG D. A game-theoretic method for cross-layer stochastic resilient control design in CPS. International Journal of Systems Science, 2018, 49(4): 677-691.

[36] CHEN Z, LIU J, LI G, et al. Composite time-consistent multiperiod risk measure and its application in optimal portfolio selection. TOP, 2016, 24(3): 515-540.

[37] LV J, RONG J. Virtualization security risk assessment for enterprise cloud services based on stochastic game nets model. IET Information Security, 2018, 12(1): 7-14.

[38] HE L, CHEN Y, ZHAO H, et al. Game-based analysis of energy-water nexus for identifying environmental impacts during Shale gas operations under stochastic input. Science of the Total Environment, 2018, 627: 1585-1601.

[39] JIANG H, SHANBHAG U V, MEYN S P. Distributed computation of equilibria in misspecified convex stochastic Nash games. IEEE Trans. on Automatic Control, 2018, 63(2): 360371.

[40] LIANG S, YI P, HONG Y. Distributed Nash equilibrium seeking for aggregative games with coupled constraints. Automatica A Journal of IFAC the International Federation of Automatic Control, 2017, 85: 179-185.

[41] ITO Y, MIYAUCHI A, ODA H. Active surveillance as the initial course of action in low-risk papillary microcarcinoma. MANCINO A T, LAWRENCE T K ed. Management of differentiated thyroid cancer. New York: Springer International Publishing, 2017.

[42] DARIO P, BASILIO G, FRANCESCA P, et al. Nash and wardrop equilibria in aggregative games with coupling constraints. IEEE Trans. on Automatic Control, 2018: 64(4): $1374-1388$.

\section{Biographies}

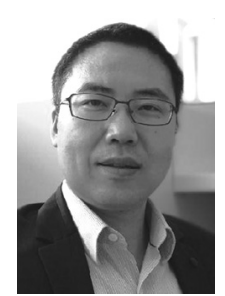

CHEN Chao was born in 1977. He received his M.S. and Ph.D. degrees from National University of Defense Technology in 2003 and 2007. He is now an associate research fellow at National University of Defense Technology. His research interests include task assignment, mission planning, and the COA.

E-mail: changshacc@163.com

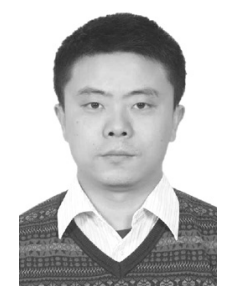

DU Zhengjun was born in 1982 . He received his M.S. and Ph.D. degrees from National University of Defense Techenology in 2008 and 2013. He is now an engineer in Unit 61683 of the PLA. His research interests include analysis of the system of systems and operation planning.

E-mail: outstandingdzj@163.com 


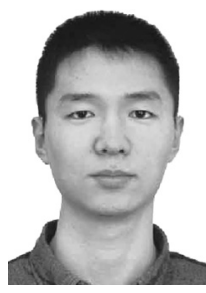

LIANG Xingxing was born in 1992. He received his B.Sc. degree from National University of Defense Technology, Changsha, Hunan, China, in 2014. He received his M.S. degree in military training from National University of Defense Technology in 2016. He is currently pursuing his Ph.D. degree in management science and engineering at $\mathrm{Na}-$ tional University of Defense Technology. His current research interests include task assignment and intelligence planning. E-mail: doublestar_1@163.com

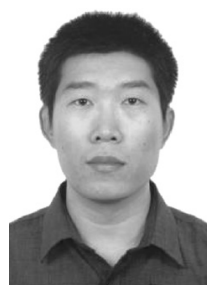

SHI Jianmai was born in $1980 . \mathrm{He}$ is an associate research fellow in National University of Defense Technology, Changsha, Hunan, China, and a researcher of Science and Technology on Information Systems Engineering Laboratory. His main research interests include task assignment, mission planning and supply chain management.

E-mail: jianmaishi@gmail.com

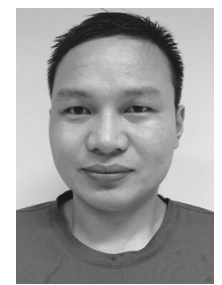

ZHANG Hao was born in 1983. He received his M.S. and Ph.D. degrees from National University of Defense Technology. He is now a lecturer at Naval Command College. His research interests include complex networks and automated planning.

E-mail: zhanghaocs@163.com 